\title{
El Coste Medico de la Depresión
}

\section{The Medical Cost of Depression}

\author{
Francisco Javier Albino-Rodríguez ${ }^{a}$, Rosario Barrera-Gálvez ${ }^{b}$, José Arias-Rico ${ }^{c}$, Claudia \\ Teresa Solano-Pérez ${ }^{d}$, Olga Rocio Chávez-Flores ${ }^{e}$, Gwendolyne Samperio-Pelcastre ${ }^{f .}$
}

\begin{abstract}
:
La depresión es un trastorno mental frecuente, caracterizado por la presencia de tristeza, entre otros síntomas que tiene una gran influencia y presencia en pacientes que han sufrido, han sido diagnosticados o superado una enfermedad, ya sea parcial o crónica. Que tiene una gran influencia en el desarrollo o sufrimiento de la depresión en tales situaciones. El objetivo es determinar cómo este evento afecta o perjudica a los pacientes y cómo se enfrentan a esta situación mental, para poder hacer frente a su enfermedad y recuperar la homeostasis de su salud óptima.
\end{abstract}

Keywords:

Depresión, enfermedad, sufrimiento, diagnóstico

\section{Resumen:}

Depression is a frequent mental disorder, characterized by the presence of sadness, among other symptoms, which has a great influence and presence in patients who have suffered, have been diagnosed or overcome a disease, either partial or chronic. . Which has a great influence on the development or suffering of depression in such situations. The objective is to determine how this event affects or damages the patients and how they face this mental situation, in order to cope with their illness and to be able to recover the homeostasis of their optimal health.

Palabras Clave:

Depression, illness, suffering, diagnosis

\section{Introducción}

La presente investigación, en base al subtema "El Coste Medico de la Depresión", del libro Inteligencia Emocional de Daniel Goleman, el cual ha desarrollado ciertas

\footnotetext{
${ }^{a}$ Universidad Autónoma del Estado de Hidalgo, Instituto de Ciencias de la Salud Correo electrónico: al413883@uaeh.edu.mx

${ }^{\mathrm{b}}$ Autor de Correspondencia, Universidad Autónoma del Estado de Hidalgo, Instituto de Ciencias de la Salud, https://orcid.org/0000-00021949-5424, Email: rosario_barrera@uaeh.edu.mx

Universidad Autónoma del Estado de Hidalgo, Instituto de Ciencias de la Salud, https://orcid.org/0000-0003-0219-0410, Email: josearias.rico@hotmail.com

${ }^{d}$ Universidad Autónoma del Estado de Hidalgo, Instituto de Ciencias de la Salud, https://orcid.org/0000-0003-4648-981X, Email: claudia_solano@uaeh.edu.mx

e Universidad Autónoma del Estado de Hidalgo, Instituto de Ciencias de la Salud, https://orcid.org/0000-0001-9479-9834, Email: ofloresc@uaeh.edu.mx

${ }^{\text {f }}$ Universidad Autónoma del Estado de Hidalgo, Instituto de Ciencias de la Salud, https://orcid.org/0000-0002-3663-6241, Email: gwendyuni@hotmail.com
} 
cuestiones, con el objetivo de determinar; que es a depresión, cual es la influencia de la depresión en una enfermedad, cuales son las causas de la misma, sus efectos, el cómo evitar la presencia de este estado mental, el cómo tratarlo, etc.

Donde Goleman habla que la depresión desempeña un papel importante dentro de las condiciones clínicas, favoreciendo al empeoramiento de una enfermedad, por lo cual es necesario que dichos pacientes reciban un óptimo tratamiento para este padecimiento. Donde de igual forma este padecimiento se puede lograr confundir en ocasiones con otro tipo de patologías a causa de su diversa sintomatología, por lo cual es necesario el poder establecer un correcto diagnóstico médico.

Determinando de igual forma que los pacientes que padecen esta condición están más predispuestos a no colaborar en el tratamiento de su enfermedad, fomentando el riesgo de su enfermedad que lo afecta.

\section{Desarrollo}

Toda enfermedad y el mismo hecho de enfermar tienen aspectos psicológicos y sociales los cuales influyen en la aparición, desarrollo, manifestación, curso y pronóstico, de una enfermedad por lo que es muy importante tratar de establecer relaciones entre aspectos psicológicos, sociales y biológicos como desencadenantes de una enfermedad, más que una relación única de causa-efecto.

En su estado de funcionamiento idóneo, el organismo mantiene un equilibrio homeostático, cuya alteración prolongada se acompaña de manifestaciones sintomáticas de orden fisiológico, biológico y/o psicológico.

Según la OMS (2017) "La depresión es un trastorno mental frecuente, que se caracteriza por la presencia de tristeza, pérdida de interés o placer, sentimientos de culpa o falta de autoestima, trastornos del sueño o del apetito, sensación de cansancio y falta de concentración." A partir de ello se puede establecer que es un trastorno el cual afecta de manera psicológica y física a las personas las cuales son susceptibles a padecerla de acuerdo a las diversas circunstancias que lo rodean o viven, teniendo una incidencia en una diversa sintomatología que en ocasiones puede pasar inadvertida o suele confundirse con otra patología de sintomatología similar, y esta de igual forma puede presentarse de manera crónica o recurrente la cual puede, interferir en el desempeño laboral, escolar al igual que la capacidad para afrontar la vida diaria (enfermedades, preocupaciones, etc.).

Según López lbor (2007) "Cuando se enferma se experimenta una serie de reacciones emocionales ante la enfermedad que el médico debe considerar, entre las que destacan la personalidad del paciente, edad, enfermedad, familia, amigos, hospital, personalidad del médico y demás profesionales de salud". A partir de ello es necesario poder llevar a cabo una correcta valoración de los pacientes por parte del personal de salud: médicos, enfermeras, etc, con la finalidad de determinar el estado de salud físico y emocional, con el objetivo de determinar el padecimiento concreto y determinar el tratamiento adecuado a sus padecimientos físicos y psicológicos, promoviendo una correcta homeostasis del organismo.

Esto es condicionado acorde a cada individuo en la manera que reacciona tras ser ingresado en un hospital, dependiendo la naturaleza de la enfermedad, de su personalidad, y de su situación vital. Entre los pacientes algunos interpretan al hospital como un lugar y/o ambiente seguro del que van a obtener cuidado y alivio en sus síntomas mientras otros demuestran una idea del cómo un lugar que aumenta su ansiedad e inquietud.

Según San Molina (2010) "Es una enfermedad de la mente y cuerpo, donde las personas tienden a padecer síntomas físicos y psicológicos", dentro de lo cual es importante resaltar padecimientos psicológicos tales como: tristeza, desinterés, ansiedad, vacío emocional, pensamientos negativos, problemas de memoria, delirios, alucinaciones e ideas de suicidio. Por otra parte, contamos con sintomatología física tal como lo es: insomnio, hipersomnio, fatiga, agitación, enlentecimiento mental y fisco, disminución de apetito, pérdida de peso.

Debido a la presencia de dicha sintomatología a causa de la depresión es necesaria la intervención de personal especializado en este tipo de trastornos mentales ya que dicho cuadro clínico puede ser a causa de su estado de salud del paciente (enfermedad parcial o crónico degenerativa), para lo cual es necesario obtener un adecuado y óptimo diagnóstico, para determinar el tratamiento e intervenciones apropiadas para el paciente con el objetivo de restablecer su estado de salud optimo el cual le permitirá reincorporarse a sus actividades cotidianas, el afrontar su enfermedad desencadenante, lo cual de esta manera generara un bien al paciente.

Para lo cual el diagnostico de dicho padecimiento se establece a partir de una valoración que se basa a partir de los siguientes factores:

- La información aportada por el paciente y familiares (anamnesis)

- La sintomatología del paciente

- La exploración física del paciente.

- Determinar la presencia de una enfermedad parcial o crónica.

Con el objetivo de establecer un diagnostico concreto y a partir de ello llevar acabo las intervenciones 
correspondientes. La prevalencia de depresión es significativamente más alta en poblaciones en contacto médico, como es el caso de los servicios de atención primaria.

Dentro de los factores desencadenantes de la depresión en base a un estado de salud se encuentran relacionados: los altos niveles de ansiedad o estrés, la personalidad, el uso de fármacos, alteraciones de los neurotransmisores cerebrales, el ambiente social y laboral.

De igual forma se sabe que este padecimiento tiene una mayor incidencia dentro de los pacientes que padecen ciertas enfermedades tales como: Cáncer, enfermedad coronaria, diabetes, epilepsia, esclerosis múltiple, derrame cerebral, enfermedad de Alzheimer, VIH o SIDA, Parkinson, lupus eritematoso sistémico, artritis reumatoide, cardiopatías, por lo cual es de suma importancia llevar un estricto control de dichos pacientes para de esta forma evitar complicaciones o defunciones acorde a su patología.

Según Harrison (2013) “El tratamiento de la depresión va de la mano de los medicamentos antidepresivos que constituyen la base del tratamiento, aunque el tratamiento combinado con psicoterapia mejora el pronóstico." Con ello podemos establecer que la depresión es un padecimiento que se pude tratar a partir del uso de fármacos como lo son los antidepresivos, los ansiolíticos (tranquilizantes) y se complementan con las sesiones terapéuticas para obtener mejores resultados.

Por otra parte, Harrison (2013) menciona otro tratamiento para la depresión crónica o letal donde "El tratamiento electroconvulsivo en general se reserva para la depresión potencialmente letal que no responde a la farmacoterapia o para los pacientes en quienes el empleo de antidepresivos tiene contraindicaciones médicas". Por cual se puede atribuir que acorde a los niveles de depresión que presenten los pacientes dentro de un ambiente hospitalario o extrahospitalario se determinara la terapia adecuada acorde a la agudez de su diagnóstico.

Casi cualquier clase de medicamento puede desencadenar o agravar una depresión. Por mencionar algunos los antihipertensivos, los anticolesterolémicos y los antiarrítmicos son detonantes comunes de síntomas depresivos. Entre los fármacos antihipertensivos, los bloqueadores $\beta$ adrenérgicos $y$, en menor grado, los antagonistas de los conductos del calcio tienen más probabilidades de ocasionar una depresión del estado de ánimo, esto acorde a que patología valla dirigido cada uno de estos fármacos que contribuyen a que el paciente desarrolle depresión a causa de una condición patológica que lo aqueje, sea de carácter parcial o crónico degenerativa.

\section{Conclusión}

A partir de esta indagación se puede establecer que la depresión es una patología que se presenta de manera recurrente dentro de un ambiente hospitalario, la cual se atribuye al desarrollo o presencia de patologías, debido al diagnóstico o pronóstico de ellas, lo cual conlleva a que los enfermos tiendan a padecerla, e incluso sea un factor negativo el cual influya en su mejoramiento, sanción o superación de su enfermedad.

Al igual interfiere el desarrollo o el ambiente en el cual se encuentra el enfermo, ya que este condiciona al mejoramiento o empeoramiento de su padecimiento, debido a esto es necesario el fomento de relaciones sociales con familia, médicos, enfermeras, amigos, con el objetivo de reconfortar y aumentar el estado anímico de los pacientes. Ya que este es un factor de suma importancia dentro de la estabilidad emocional de los pacientes de las diversas enfermedades.

Por lo cual es necesario que dentro de las instituciones de atención médica, se lleve a cabo una implementación de diagnóstico de este tipo de patología, ya que de esta manera se proporciona un apoyo extra a los pacientes, con la finalidad de evitar un empeoramiento de su estado de salud, el cual sea perjudicial para su salud, debido a que la depresión se puede clasificar en leve y crónica, las cuales tienen una importante influencia y acorde a ellas se determinara la intensidad de su tratamiento.

De igual forma el tener un óptimo tratamiento y pronóstico de la enfermedad interfiere en la mejora de los individuos que los aqueja, contemplando tanto el tratamiento farmacológico de la patología que aqueje al paciente al igual que el tratamiento farmacológico antidepresivo y terapias adecuadas para su sanción, debido a que incluso la medicina convencional en ocasiones influye en el desarrollo de este padecimiento.

\section{Referencias}

[1] Long, L. D. (2013). Harrison. Manual médico (18.a ed.). McGraw Hill.

[2] López Ibor, M. I. (2007). Ansiedad y depresión, reacciones emocionales frente a la enfermedad. Anales De Medicina Interna, 24(5), 209-211. http://scielo.isciii.es/pdf/ami/v24n5/editorial.pdf

[3] San Molina, L. (2010). Comprender la Depresión (1.a ed.). Amat Editorial.

[4] World Health Organization. (2017, 23 febrero). Depresión. Organización Mundial de la Salud. https://www.who.int/topics/depression/es/ 